\title{
Knee pain in saphenous nerve schwannoma
}

\author{
Case report
}

\author{
Roberto Gazzeri, M.D., ${ }^{1}$ Gianni M. Refice, M.D. ${ }^{1}$ Marcelo Galarza, M.D., ${ }^{1}$ \\ Massimiliano Neroni, M.D., ${ }^{1}$ Stefano Esposito, M.D., ${ }^{1}$ AND Giovanni Gazzeri, M.D. ${ }^{2}$
}

${ }^{1}$ Department of Neurosurgery, San Giovanni-Addolorata Hospital; and ${ }^{2}$ Department of Neurosurgery, San Filippo Neri Hospital, Rome, Italy

\begin{abstract}
$\checkmark$ The differential diagnosis of anteromedial knee pain is extensive and can be associated with meniscal tear, medial collateral ligament sprain, or pes anserinus bursitis. An association between knee pain and a peripheral nerve sheath tumor has rarely been reported. The authors describe the case of a 44-year-old man with a saphenous nerve schwannoma who presented with vague pain in the medial aspect of his knee mimicking the clinical presentation of a meniscal tear.
\end{abstract}

KeY WoRdS • knee pain • peripheral nerve sheath tumor • saphenous nerve • schwannoma

\section{$\mathrm{T}$}

HE DIFFERENTIAL DIAGNOSIS of knee pain is extensive: unexplained anteromedial knee pain can be associated with minor meniscal tear, medial collateral ligament sprain, or pes anserinus bursitis.,3 A patient harboring nerve schwannomas presenting with vague pain in the medial aspect of the knee mimicking a meniscal tear is a very rare event that should be considered by physicians examining patients with knee pain.

\section{Case Report}

History and Examination. This 44-year-old man presented to our department with a 4-month history of pain in his left knee following a minor trauma. He reported vague knee pain with a tender area over the anteromedial aspect of the left knee associated with paresthesias and laterally radiating pain. He experienced this pain while in a semisitting position, and he had no relief with analgesic medications. He had been examined by several physicians who diagnosed a medial meniscal tear. Previous plain $\mathrm{x}$ ray films of the left knee were nondiagnostic, and MR images showed normal meniscal anatomy.

A neurological examination revealed intact motor function and paresthesias on the anteromedial aspect of the knee. Signs of mild atrophy of the vastus medialis oblique muscle of the quadriceps were present. Knee palpation

Abbreviations used in this paper: $\mathrm{MR}=$ magnetic resonance. showed tenderness at the medial joint line. No knee joint effusion was present. The McMurray Test to determine the presence of a medial meniscus tear (the tibia is rotated externally, and the knee is extended from maximal flexion to approximately $90^{\circ}$ ) was positive, causing pain. A positive Tinel sign over the anteromedial aspect of the knee was also present. On physical examination, a palpable rounded mass in a deep location in the caudal aspect of the left thigh was noted, and deep pressure of the lesion elicited pain that radiated along the anteromedial aspect of the knee. Magnetic resonance images of the left thigh showed a well-defined, rounded, enhancing mass lesion measuring $2.2 \mathrm{~cm}$ in diameter and lying medial to the sartorius and gracilis muscles (Fig. 1). Electromyography was nondiagnostic.

Operation. With the patient in a state of general anesthesia, a $15-\mathrm{cm}$ skin incision was made on the medial compartment of the thigh from the medial epicondyle of the femur and centered on the lesion. After incision of the crural fascia between the vastus medialis and sartorius muscles, the saphenous nerve with the encapsulated mass was exposed. Under an operating microscope, the lesion was easily dissected from the saphenous nerve fascicles using a Rothon dissector.

Postoperative Course. Macroscopically, the tumor appeared encapsulated, shiny, and yellow (Fig. 2). The histological diagnosis was benign schwannoma. The patient was discharged on the 2 nd postoperative day, al- 


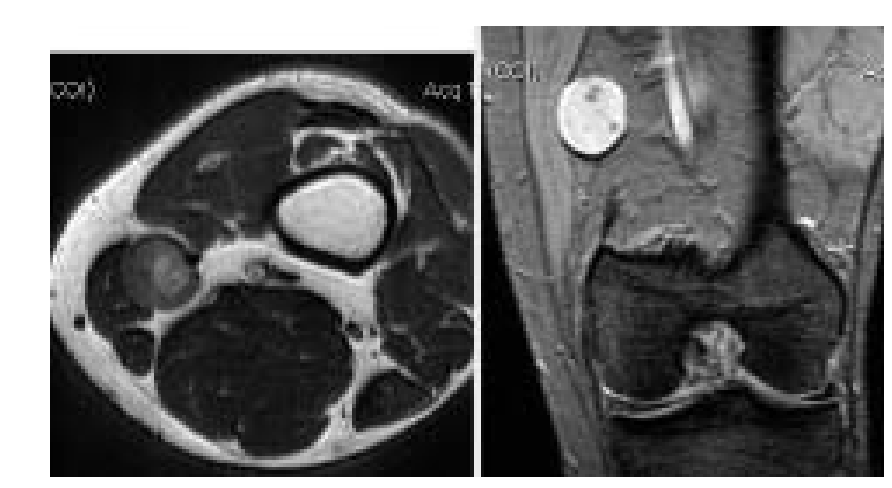

FIG. 1. Preoperative axial contrast-enhanced T1-weighted MR image (left) and coronal T2-weighted MR image (right) demonstrating a saphenous nerve schwannoma.

though he had numbness in the left saphenous nerve distribution. By the 4-month follow-up he was symptom free, with no pain or sensory disturbances.

\section{Discussion}

We here report on a case of chronic knee pain caused by a saphenous nerve benign schwannoma. The differential diagnosis of knee pain is extensive but can be narrowed with a detailed history, physical and neurological examinations, and appropriate use of MR imaging. It is important to note the characteristics of pain-its onset, location (anterior, medial, lateral, or posterior knee), duration, and quality. Furthermore, aggravating and alleviating factors must be addressed. Anteromedial knee pain can be associated with a meniscal tear, a medial collateral ligament sprain, or pes anserinus bursitis. A meniscal tear can occur in association with a prolonged degenerative process, particularly in a patient with a knee deficient in anterior cruciate ligament. Such a patient usually reports recurrent knee pain and episodes of catching of the knee joint with squatting or twisting of the knee. On physical examination, mild effusion may be noticeable, with tenderness at the medial or lateral joint line. Atrophy of the vastus medialis oblique portion of the quadriceps muscle can be present. The McMurray Test may be positive, but a negative test does not eliminate the possibility of a meniscal tear. ${ }^{2,3}$ The patient in the present case was examined by several physicians, all of whom had diagnosed a minor medial meniscal tear.

Knee pain can also be associated with traumatic or surgical injury of a branch of the saphenous nerve, ${ }^{13}$ which is the largest cutaneous branch of the femoral nerve. It descends lateral to the femoral artery into the adductor canal and proceeds vertically along the medial side of the knee behind the sartorius muscle, where it gives off an infrapatellar branch that pierces the fascia lata between the sartorius and gracilis muscles to become subcutaneous. ${ }^{1}$ In the medial aspect of the knee, fine branches of the saphenous nerve are distributed where the nerve becomes cutaneous, communicating with anterior cutaneous branches of the femoral nerve. ${ }^{1,12}$ Schwannoma of the saphenous nerve has seldom been reported, although it is usually associated with neurofibromatosis Type $1 .^{4,5,7,9,10}$

Schwannomas are rare, benign, often solitary, slow-

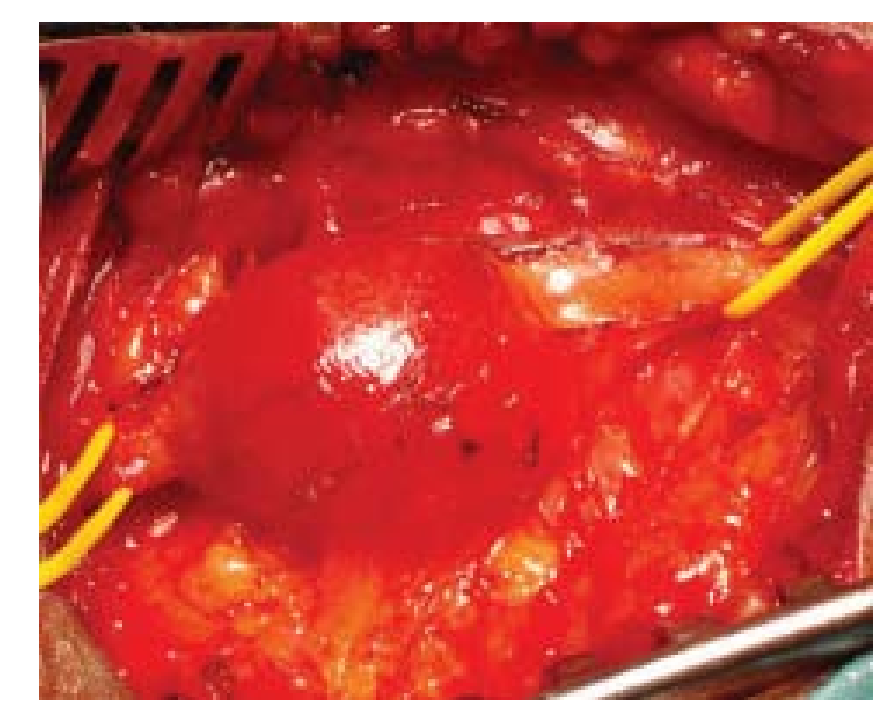

FIG. 2. Intraoperative photograph showing the saphenous nerve with an encapsulated lesion.

growing nerve sheath tumors. ${ }^{6,8,11}$ They are most often located in the brachial plexus $(39 \%)$, followed by a slight preponderance in the upper limbs $(30 \%)$ compared with their appearance in the lower extremities (24\%). ${ }^{7}$ They are usually clinically silent and thus any presentation, as in our patient, usually makes diagnosis difficult. Symptoms appear years after lesion formation, unless the schwannoma is compressed by external forces or anatomical confinement. Tumor compression can evoke pain at the site of the lesion, or pain may radiate along the course of the involved nerve. We suggest that in the featured case, hyperextension and hyperflexion of the leg could have provoked compression between the muscular layers of the lesion and nerve fibers that innervated the medial aspect of the knee, leading to a misdiagnosis of medial meniscal disease. Treatment of symptomatic schwannoma involves resection with little or no injury to the parent nerve. As in the patient in our case, electromyography studies are not useful in the diagnosis of these lesions because nerve conduction is rarely altered.

This present case is of particular interest given the rarity of the lesion localization along the course of the saphenous nerve (the other few cases of saphenous nerve schwannoma have been localized in a more proximal or caudal position) and the atypical symptoms that the tumor induced. An association between knee pain and a peripheral nerve sheath tumor has rarely been reported. One previous case of anterior knee pain associated with a schwannoma of the saphenous nerve localized in the Hunter canal has been described. ${ }^{5}$ The present case represents the first of a saphenous nerve schwannoma mimicking meniscal tear symptoms to be reported in the literature. Although rare, saphenous nerve schwannoma can be suspected in patients suffering knee pain with meniscal symptoms and a nondiagnostic MR image of the knee joint.

\section{References}

1. Berry M, Bannister LH, Standring SM: Nervous system, in Williams PL (ed): Gray's Anatomy, ed 38. Edinburgh: Churchill Livingstone, 1995, pp 1279-1282 
2. Calmbach WL, Hutchens M: Evaluation of patients presenting with knee pain: part I. History, physical examination, radiographs, and laboratory tests. Am Fam Physician 68:907-912, 2003

3. Calmbach WL, Hutchens M: Evaluation of patients presenting with knee pain: part II. Differential diagnosis. Am Fam Physician 68:917-922, 2003

4. Chatillon CE, Guiot MC, Corriveau MM, Jacques L: Superficial femoral vein invasion by a benin neurofibroma in a non-neurofibromatosis patient: case report. Neurosurgery 58:E997, 2006

5. Edwards JC, Green T, Riefel E: Neurilemoma of the saphenous nerve presenting as pain in the knee. A case report. J Bone Joint Surg Am 71:1410-1411, 1989

6. Halliday AL, Sobel RA, Martuza RL: Benign spinal nerve sheath tumors: their occurrence sporadically and in neurofibromatosis types 1 and 2. J Neurosurg 74:248-253, 1991

7. Kim D, Murovic JA, Tiel RL, Moes G, Kline D: A series of 397 peripheral neural sheath tumors: 30-year experience at Louisiania State University Health Sciences Centre. J Neurosurg 102:246-255, 2005

8. Kransdorf MJ: Benign soft-tissue tumors in a large referral popu- lation: distribution of specific diagnoses by age, sex, and location. AJR Am J Roentgenol 164:395-402, 1995

9. Ogose A, Hotta T, Morita T, Otsuka H, Hirata Y: Multiple schwannomas in the peripheral nerves. J Bone Joint Surg Br 80:657-661, 1998

10. Pearce JM: Neurofibromatosis. J Neurol Neurosurg Psychiatry 74:384, 2003

11. Rubinstein LJ: The malformative central nervous system lesions in the central and peripheral forms of neurofibromatosis. A neuropathological study of 22 cases. Ann N Y Acad Sci 486: 14-29, 1986

12. Sunderland S (ed): Nerves and Nerve Injuries. New York: Churchill and Livingstone 1978, pp 999-1006

13. Tennent TD, Birch NC, Holmes MJ, Birch R, Goddard NJ: Knee pain and the infrapatellar branch of the saphenous nerve. J R Soc Med 91:573-575, 1998

Manuscript submitted February 7, 2007.

Accepted April 26, 2007.

Address reprint requests to: Roberto Gazzeri, M.D., Via O. Tommasini 13, 00162, Roma, Italy. email: robertogazzeri@gmail.com. 